\title{
Effect of Perioperative CEA and CA24-2 on Prognosis of Early Resectable Pancreatic Ductal Adenocarcinoma
}

\author{
Xiaojie $\mathrm{Li}^{1}$, Shengnan $\mathrm{Li}^{1}$, Lili Liu ${ }^{2}$, Jiahui Hong ${ }^{2}$, Tiansuo Zhao ${ }^{1}$, Chuntao Gao $^{1 凶}$ \\ 1. Department of Pancreatic Cancer, Tianjin Medical University Cancer Institute and Hospital, National Clinical Research Center for Cancer, Key Laboratory \\ of Cancer Prevention and Therapy, Tianjin's Clinical Research Center for Cancer, Tianjin, China, 300060 \\ 2. Department of Nutrition and Food Hygiene, School of Public Health, Tianjin Medical University, Tianjin, China, 300060 \\ $\square$ Corresponding author: Chuntao Gao, Department of Pancreatic Cancer, Tianjin Medical University Cancer Institute and Hospital, National Clinical Research \\ Center for Cancer, Key Laboratory of Cancer Prevention and Therapy, Tianjin's Clinical Research Center for Cancer, Tianjin, China, 300060. (E-mail: \\ gaochuntao@tjmuch.com).
}

(c) The author(s). This is an open access article distributed under the terms of the Creative Commons Attribution License (https://creativecommons.org/licenses/by/4.0/). See http://ivyspring.com/terms for full terms and conditions.

Received: 2019.02.01; Accepted: 2019.09.16; Published: 2020.01.01

\begin{abstract}
Patients with resectable pancreatic ductal adenocarcinoma (PDAC) show differential prognosis after radical resection. Currently, cancer grading and surgical criteria depend heavily on imaging and anatomical diagnosis. It's essential to set up a model with reliable prognostic factors during the perioperative period to assess prognosis in PDAC patients. In this study, 103 patients diagnosed with PDAC who underwent radical resection were recruited. The predictive value of preoperative carcinoembryonic antigen (CEA), postoperative CA24-2 and the combination of two for overall survival (OS) were evaluated. Both pre-CEA and post-CA24-2 were found to be independent prognostic factors for OS according to multivariate analyses. Kaplan-Meier analysis revealed that CEA and CA24-2 as well as the combination of two were correlated with poor OS. In addition, patients with both markers elevated have worse prognosis than patients with either pre-CEA or post-CA24-2 elevated. Thus, we concluded that the combination of CEA and CA24-2 can be used as a prognostic factor for stage I and II resectable PDAC patients.
\end{abstract}

Key words: Pancreatic cancer, Radical resection, CEA antigen, CA24-2 antigen, Prognosis

\section{Introduction}

Pancreatic ductal adenocarcinoma (PDAC) is the fourth leading cause of cancer mortality worldwide with a five-year survival rate of approximately $7.2 \%$ [1]. Up to date, surgery is the only effective treatment option for PDAC, which can increase the 5-year survival rate by $20 \%-25 \%$ [2, 3]. However, only $15 \%-20 \%$ of patients diagnosed during early cancer stage are eligible for radical surgery. Furthermore, this invasive technique does not guarantee a cure, and sometimes may lead to poor response to chemotherapy [4].

Currently, surgical guideline of PDAC still relies on imaging and tumor anatomy. Studies of postoperative PDAC prognosis have shown that clinical pathological features such as tumor size, lymph node metastasis, neurological invasion, distant metastasis, and vascular invasion may influence disease-specific survival(DSS), disease-free survival (DFS), and overall survival(OS) [5, 6, 7]. Serological indicators such as LDH, ALB, ALP, NLR and CRP affect prognosis of patients with stage III and IV PDAC characterized by distant metastasis and local progression [8]. While serum CA19-9, CA12-5 and CEA have been reported as markers of prognosis for PDAC at all stages [9]. Moreover, TNM Classification of Malignant Tumors may also affect treatment plan and prognosis of patients [10]. Overall there are no reliable biological markers to guide adjuvant therapy and determine prognosis of PDAC. In this study, our retrospective cohort analysis investigated clinical data, laboratory tests and pathological diagnosis before and after PDAC resection. We aim to identify 
possible markers related to PDAC radical resection in order to provide a basis for clinical evaluation of patient prognosis.

\section{Materials and Methods}

\section{Patients}

103 patients diagnosed with PDAC and underwent radical resection in Tianjin Medical University Cancer Institute and Hospital from 2011 to 2015 were recruited for our study. Eligibility for radical resection was evaluated according to NCCN guideline [11]. Pancreaticoduodenectomy or spleen and distal pancreatectomy were performed based on tumor location and growth. Patients underwent admission assessments including chest X-ray, abdominal CT or MR, and baseline observations were recorded. Postoperative tissue biopsy confirmed pancreatic ductal adenocarcinoma diagnosis.

All patients underwent postoperative adjuvant chemotherapy within 1 or 2 months after surgery. We conducted regular telephone follow-up of all enrolled patients. The exclusion criteria for this study included: 1. Preoperative assessment was not found, but intraoperative pathology diagnosed patients with distant metastases. 2. Non-R0 resection of the patient. 3. Patients who died within 1 month after surgery. 4 . Patients who did not undergo standard adjuvant chemotherapy after surgery. 5. Patients who were unwilling to undergo research or unable to follow up. This study has passed the ethical review of the Ethics Committee of the Tianjin Medical University Cancer Institute and Hospital.

\section{Histopathological examination}

Tumor tissues used for diagnostics were all collected during surgery. The histopathological criteria used in this study included: tumor size, tumor location, carcinoma cell embolism, lymph node metastasis, distant metastasis, perineural invasion, pancreatic capsule invasion, and presence of R0 resection. TNM staging of PDAC were determined based on histopathology in accordance with the American Joint Commission on Cancer (AJCC) [11].

\section{Laboratory testing}

Serum CA19-9, CEA, CA24-2, LDH, ALP, TBIL, DBIL, ALB, and NLR were collected within 7 days before surgery. ALB was collected again 7 days after surgery. CA19-9, CEA and CA24-2 were collected 30 days after surgery. The cut-off values were defined as the upper limits of normal CA19-9, CEA, CA24-2, LDH, ALP, TBIL, DBIL, ALB, and the lower limits of normal ALB. We set the cutoff value of NLR to 5 .

\section{Study design and statistical analysis}

All statistical analyses were performed using SPSS software (version20.0). Data between groups were compared using Pearson $\chi^{2}$ and Fisher's exact, and the inter-group OS was compared using non-parametric statistics. Univariate COX regression was used to screen for potential prognostic factors, and $\mathrm{p}$ value $\leq 0.20$ was used for multivariate COX regression to calculate $H R$ and determine independent prognostic factors. Survival analysis was performed using the Kaplan-Meier method. Survival period is the interval between the day of surgery and death or loss of follow-up. Statistical significance was defined as $\mathrm{p}$ value $\leq 0.05$.

\section{Results}

\section{Patient characteristics}

This study included 103 patients, 65 males (63.1\%) and 38 females (36.9\%), with PDAC who were admitted to the Tianjin Medical University Cancer Institute and Hospital from 2011 to 2015. Preoperative evaluation confirmed the presence of resectable pancreatic cancer. The median age of patients was 60 (range from $42-80)$. There were 65 cases $(63.1 \%$ ) of tumor located in the head and uncinate process of pancreas, and 38 cases (36.9\%) were located in the pancreatic body and tail. Based on the pathological TNM staging system, 43 patients $(41.7 \%)$ were in Stage I and 60 patients (58.3\%) were in Stage II. There were 63 patients $(61.2 \%)$ with tumor size smaller than $4 \mathrm{~cm}$ and 40 patients (38.8\%) with size greater than 4 $\mathrm{cm}$. A total of 28 cases (27.2\%) exhibited local lymph node metastasis. In addition, there were 18 cases $(17.5 \%)$ with presence of carcinoma cell emboli, 40 cases (38.8\%) with perineural invasion, 81 cases (78.6\%) with pancreatic capsule invasion, and 23 cases $(22.3 \%)$ with vascular invasion. The general characteristics, including laboratory tests, are summarized in Table 1.

\section{Independent prognosis factors for resectable PDAC with stage I and II}

We initially selected 28 risk factors that may affect the overall survival of patients with PDAC after radical surgery based on basic clinical information, pathological finding and laboratory results of patients. Univariate COX analysis identified 7 candidate indicators for poor prognosis, including age (HR=0.687, $\mathrm{p}=0.195)$, preoperative CA19-9 $(\mathrm{HR}=1.454$, $\mathrm{p}=0.195)$, preoperative CEA $(\mathrm{HR}=2.348, \mathrm{p}=0.001)$, preoperative DBIL $(\mathrm{HR}=0.654, \mathrm{p}=0.107)$, postoperative CA19-9 $(\mathrm{HR}=1.636, \mathrm{p}=0.025)$, postoperative CEA $(\mathrm{HR}=2.187, \mathrm{p}=0.012)$, and postoperative CA24-2 ( $H R=1.849, p=0.007)$, which were further evaluated by 
multivariate COX regression analysis. From that, the forward and backward LR methods verified that elevated preoperative CEA $(\mathrm{HR}=2.212, \mathrm{p}=0.002$, $\mathrm{p}<0.05)$ and postoperative CA24-2 $(\mathrm{HR}=1.731$, $p=0.017, p<0.05)$ were independent factors of poor prognosis and OS (Table 2).

Table 1. Clinicopathologic characteristics of patients with resectable PDAC

\begin{tabular}{|c|c|c|c|c|}
\hline Characteristics & Number & Percentage (\%) & Median OS (months) & p-value \\
\hline Total & 103 & 100 & 20 & \\
\hline \multicolumn{5}{|l|}{ Clinical characteristics } \\
\hline \multicolumn{5}{|l|}{ Age (years) } \\
\hline \multicolumn{5}{|l|}{ Median (range) 60 (42-80) } \\
\hline$<65$ vs. $\geq 65$ & 81 vs. 22 & 78.6 vs. 21.4 & 20 vs. 25 & 0.183 \\
\hline Gender (Male vs. Female) & 65 vs. 38 & 63.1 vs. 36.9 & 18 vs. 21 & 0.255 \\
\hline Tumor location (Head and Uncinate process vs. Body and Tail) & 65 vs. 38 & 63.1 vs. 36.9 & 18 vs. 20 & 0.393 \\
\hline Stage (Stage I vs. Stage II) & 43 vs. 60 & 41.7 vs. 58.3 & 20 vs. 18 & 0.327 \\
\hline Primary tumor(T) (1 and 2 vs. 3 ) & 63 vs. 40 & 61.2 vs. 38.8 & 20 vs. 18 & 0.960 \\
\hline Regional lymph(N) (0 vs. 1) & 75 vs. 28 & 72.8 vs. 27.2 & 20 vs. 20 & 0.337 \\
\hline \multicolumn{5}{|l|}{ Histopathology characteristics } \\
\hline Carcinoma cell embolus (Present vs. Absent) & 18 vs. 85 & 17.5 vs. 82.5 & 21 vs. 20 & 0.332 \\
\hline Perineural invasion (Present vs. Absent) & 40 vs. 63 & 38.8 vs. 61.2 & 17 vs. 20 & 0.526 \\
\hline Pancreatic capsule invasion (Present vs. Absent) & 81 vs. 22 & 78.6 vs. 21.4 & 20 vs. 17 & 0.480 \\
\hline Vascular invasion (Present vs. Absent) & 23 vs. 80 & 22.3 vs. 77.7 & 22 vs. 18 & 0.232 \\
\hline \multicolumn{5}{|l|}{ Laboratory testing } \\
\hline Preoperative CA19-9 ( $\leq$ ULN vs. >ULN) & 18 vs. 85 & 17.5 vs. 82.5 & 21 vs. 18 & 0.182 \\
\hline Preoperative CEA ( $\leq$ ULN vs. >ULN) & 69 vs. 34 & 67.0 vs. 33.0 & 21 vs. 16 & $<0.001$ \\
\hline Preoperative CA24-2 ( $\leq$ ULN vs. >ULN) & 39 vs. 64 & 37.9 vs. 62.1 & 21 vs. 18 & 0.332 \\
\hline Preoperative LDH ( $\leq$ ULN vs. >ULN) & 83 vs. 20 & 80.6 vs. 19.4 & 18 vs. 24 & 0.446 \\
\hline Preoperative ALP ( $\leq \mathrm{ULN}$ vs. >ULN) & 46 vs. 57 & 44.7 vs. 55.3 & 20 vs. 20 & 0.650 \\
\hline Preoperative TBIL ( $\leq$ ULN vs. >ULN) & 44 vs. 59 & 42.7 vs. 57.3 & 18 vs. 20 & 0.696 \\
\hline Preoperative DBIL ( $\leq$ ULN vs. >ULN) & 27 vs. 76 & 26.2 vs. 73.8 & 17 vs. 21 & 0.097 \\
\hline Preoperative Albumin ( $\geq$ LLN vs. $<$ LLN) & 95 vs. 8 & 92.2 vs. 7.8 & 22 vs. 18 & 0.232 \\
\hline Preoperative NLR $(\leq 5$ vs. $>5)$ & 93 vs. 10 & 90.3 vs. 9.7 & 20 vs. 20 & 0.289 \\
\hline Postoperative CA19-9 ( $\leq$ ULN vs. >ULN) & 58 vs. 45 & 56.3 vs. 43.7 & 21 vs. 13 & 0.021 \\
\hline Postoperative CEA (SULN vs. >ULN) & 89 vs. 14 & 86.4 vs. 13.6 & 20 vs. 12 & 0.008 \\
\hline Postoperative CA24-2 ( $\leq$ ULN vs. >ULN) & 61 vs. 42 & 59.2 vs. 40.8 & 21 vs. 12 & 0.005 \\
\hline Postoperative Albumin ( $\geq$ LLN vs. $<$ LLN) & 52 vs. 51 & 50.5 vs. 49.5 & 20 vs. 17 & 0.361 \\
\hline
\end{tabular}

Table 2. Univariate and multivariate analysis of prognostic factors

\begin{tabular}{|c|c|c|c|}
\hline Predictor & Patients (n) & $\mathrm{HR}(95 \% \mathrm{CI})$ & p-value \\
\hline \multicolumn{4}{|l|}{ Univariate analysis } \\
\hline Age (<65 vs. $\geq 65)$ & 81 vs. 22 & $0.687(0.389-1.213)$ & 0.195 \\
\hline Gender (male vs. female) & 65 vs. 38 & $0.776(0.496-1.215)$ & 0.268 \\
\hline Tumor location (head and uncinate process vs. body and tail) & 65 vs. 38 & $0.826(0.528-1.294)$ & 0.404 \\
\hline Stage (stage I vs. stage II) & 43 vs. 60 & $1.236(0.801-1.909)$ & 0.339 \\
\hline Primary tumor(T) (1 and 2 vs. 3$)$ & 63 vs. 40 & 1.011.(0.654-1.562) & 0.961 \\
\hline Regional lymph(N) (0 vs. 1) & 75 vs. 28 & $1.264(0.773-2.066)$ & 0.350 \\
\hline Carcinoma cell embolus (present vs. absent) & 18 vs. 85 & $0.765(0.439-1.322)$ & 0.344 \\
\hline Perineural invasion (present vs. absent) & 40 vs. 63 & $1.149(0.741-1.782)$ & 0.535 \\
\hline Pancreatic capsule invasion (present vs. absent) & 81 vs. 22 & $0.834(0.499-1.395)$ & 0.490 \\
\hline Vascular invasion (present vs. absent) & 23 vs 80 & $0.740(0.446-1.228)$ & 0.244 \\
\hline Preoperative CA19-9 ( $\leq \mathrm{ULN}$ vs. $>\mathrm{ULN})$ & 18 vs. 85 & $1.454(0.826-2.561)$ & 0.195 \\
\hline Preoperative CEA ( $\leq \mathrm{ULN}$ vs. >ULN) & 69 vs. 34 & $2.348(1.437-3.835)$ & 0.001 \\
\hline Preoperative CA24-2 ( $\leq \mathrm{ULN}$ vs. $>\mathrm{ULN})$ & 39 vs. 64 & $1.236(0.797-1.919)$ & 0.344 \\
\hline Preoperative LDH ( $\leq \mathrm{ULN}$ vs. >ULN) & 83 vs. 20 & $0.813(0.471-1.403)$ & 0.457 \\
\hline Preoperative ALP ( $\leq \mathrm{ULN}$ vs. >ULN) & 46 vs. 57 & $1.102(0.716-1.696)$ & 0.658 \\
\hline Preoperative TBIL ( $\leq$ ULN vs. >ULN) & 44 vs. 59 & $1.088(0.705-1.680)$ & 0.702 \\
\hline Preoperative DBIL ( $\leq \mathrm{ULN}$ vs. >ULN) & 27 vs. 76 & $0.654(0.390-1.096)$ & 0.107 \\
\hline Preoperative Albumin ( $\geq \mathrm{LLN}$ vs. $<\mathrm{LLN}$ ) & 95 vs. 8 & $1.126(0.517-2.452)$ & 0.765 \\
\hline Preoperative NLR ( $\leq 5$ vs. $>5$ ) & 93 vs. 10 & $1.513(0.689-3.320)$ & 0.302 \\
\hline Postoperative CA19-9 ( $\leq$ ULN vs. >ULN) & 58 vs. 45 & $1.636(1.064-2.517)$ & 0.025 \\
\hline Postoperative CEA ( $\leq \mathrm{ULN}$ vs. >ULN) & 89 vs. 14 & $2.187(1.192-4.014)$ & 0.012 \\
\hline Postoperative CA24-2 ( $\leq$ ULN vs. >ULN) & 61 vs. 42 & $1.849(1.183-2.891)$ & 0.007 \\
\hline Postoperative Albumin ( $\geq$ LLN vs. $<$ LLN) & 52 vs 51 & $1.216(0.791-1.871)$ & 0.373 \\
\hline \multicolumn{4}{|l|}{ Multivariate analysis } \\
\hline Preoperative CEA ( $\leq \mathrm{ULN}$ vs. >ULN) & 69 vs. 34 & $2.212(1.351-3.623)$ & 0.002 \\
\hline Postoperative CA24-2 ( $\leq$ ULN vs. >ULN) & 61 vs. 42 & $1.731(1.103-2.717)$ & 0.017 \\
\hline
\end{tabular}


Table 3. Correlation between preoperative CEA/postoperative CA24-2 and clinicopathologic characteristics

\begin{tabular}{|c|c|c|c|c|c|c|c|c|c|c|c|}
\hline \multirow[t]{2}{*}{ Characteristics } & \multirow[t]{2}{*}{ Value } & \multicolumn{3}{|c|}{ Preoperative CEA } & \multicolumn{3}{|c|}{ Postoperative CA24-2 } & \multicolumn{4}{|c|}{ Combination of 2 factors } \\
\hline & & $\leq \mathrm{ULN}$ & $>\mathrm{ULN}$ & p-value & $\leq \mathrm{ULN}$ & $>\mathrm{ULN}$ & p-value & 0 & 1 & 2 & p-value \\
\hline \multirow[t]{2}{*}{ Age (years) } & $<65$ & 57 & 24 & 0.162 & 49 & 32 & 0.615 & 39 & 28 & 14 & 0.499 \\
\hline & $\geq 65$ & 12 & 10 & & 12 & 10 & & 8 & 8 & 6 & \\
\hline \multirow[t]{2}{*}{ Gender } & Male & 41 & 24 & 0.269 & 39 & 28 & 0.534 & 26 & 26 & 13 & 0.281 \\
\hline & Female & 28 & 10 & & 24 & 14 & & 21 & 10 & 7 & \\
\hline \multirow[t]{2}{*}{ Tumor location } & Head and Uncinate process & 41 & 24 & 0.269 & 42 & 23 & 0.145 & 31 & 21 & 13 & 0.761 \\
\hline & Body and Tail & 28 & 10 & & 19 & 19 & & 16 & 15 & 7 & \\
\hline \multirow[t]{2}{*}{ Primary tumor(T) } & 1 and 2 & 44 & 19 & 0.440 & 39 & 24 & 0.487 & 28 & 27 & 8 & 0.035 \\
\hline & 3 & 25 & 15 & & 22 & 18 & & 19 & 9 & 12 & \\
\hline \multirow[t]{2}{*}{ Regional lymph(N) } & 0 & 55 & 20 & 0.025 & 51 & 24 & 0.003 & 42 & 22 & 11 & 0.002 \\
\hline & 1 & 14 & 14 & & 10 & 18 & & 5 & 14 & 9 & \\
\hline \multirow[t]{2}{*}{ Stage } & I & 34 & 9 & 0.027 & 32 & 11 & 0.008 & 25 & 16 & 2 & 0.004 \\
\hline & II & 35 & 25 & & 29 & 31 & & 22 & 20 & 18 & \\
\hline \multirow[t]{2}{*}{ Carcinoma cell embolus } & Present & 14 & 4 & 0.284 & 11 & 7 & 0.858 & 9 & 7 & 2 & 0.618 \\
\hline & Absent & 55 & 30 & & 50 & 35 & & 38 & 29 & 18 & \\
\hline \multirow[t]{2}{*}{ Perineural invasion } & Present & 27 & 13 & 0.930 & 25 & 15 & 0.590 & 18 & 16 & 6 & 0.566 \\
\hline & Absent & 42 & 21 & & 36 & 27 & & 29 & 20 & 14 & \\
\hline \multirow{2}{*}{$\begin{array}{l}\text { Pancreatic capsule } \\
\text { invasion }\end{array}$} & Present & 52 & 29 & 0.247 & 47 & 34 & 0.635 & 36 & 27 & 18 & 0.380 \\
\hline & Absent & 17 & 5 & & 14 & 8 & & 11 & 9 & 2 & \\
\hline \multirow[t]{2}{*}{ Vascular invasion } & Present & 17 & 6 & 0.423 & 47 & 33 & 0.855 & 11 & 9 & 3 & 0.671 \\
\hline & Absent & 52 & 28 & & 14 & 9 & & 36 & 27 & 17 & \\
\hline
\end{tabular}

A

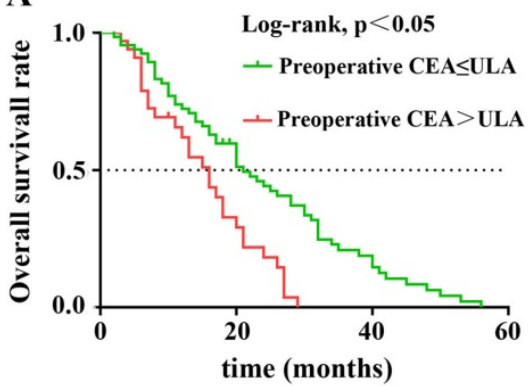

B

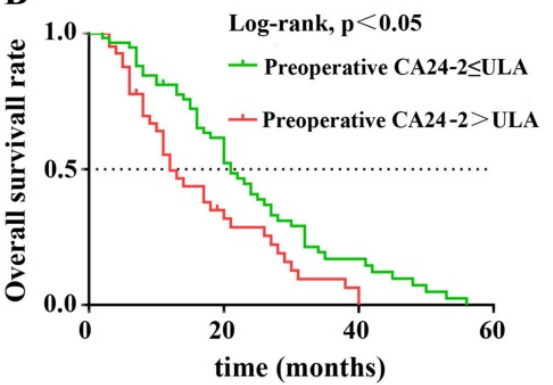

C

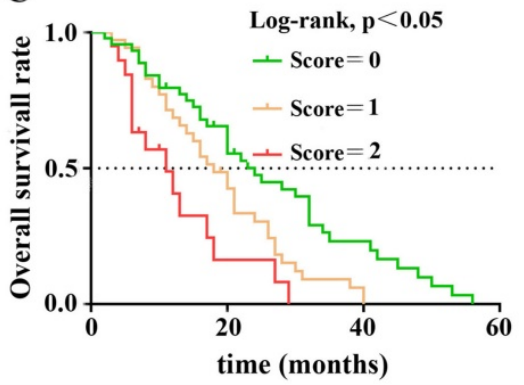

Figure 1. Kaplan-Meier overall survival curves for 103 patients with resectable PDAC stratified by (A) preoperative CEA level, (B) postoperative CA24-2 level, (C) combined score of preoperative CEA and postoperative CA24-2 levels.

\section{Preoperative CEA and postoperative CA24-2 levels correlated with clinical characteristics}

We compared inter-group relationship between preoperative CEA, postoperative CA24-2, and clinical pathological features. Elevation of either preoperative CEA or postoperative CA24-2 was given a score of 1 . Consequently, patients were divided into three groups with scores of 0 (no elevation), 1 (elevation of either CEA or CA24-2), 2 (elevation of both CEA and CA24-2). Results showed that late stage PDAC was highly correlated with increased preoperative CEA, postoperative CA24-2 and the combination of two ( $p=0.027, p=0.008$ and $p=0.004$ ). Regional lymph node invasion also correlated with postoperative CA24-2 and the combination of two $(p=0.003, p=0.002)$. Interestingly, primary tumor $(\mathrm{T})$ was only correlated with the combination of both elevated CEA and CA24-2 $(p=0.035)$ (Table 3).
Combined scores of postoperative CA24-2 and preoperative CEA were associated with postoperative prognosis of PDAC

In nonparametric tests, we found that patients with either elevated preoperative CEA or postoperative CA24-2 predicted a lower OS after surgery ( 21 vs. 16 months, $p=0.001 ; 21$ vs. 12 months, $p=0.005$ ) When preoperative CEA and postoperative CA24-2 were combined, patient OS decreased further $(p<0.001)$ suggesting an increase in both factors were more detrimental to patient survival. The KaplanMeier method was used to compare the effects of preoperative CEA and postoperative CA24-2 on the OS between groups (Figure $1 \mathrm{~A}$ and $\mathrm{B}$ ). The combined scores of preoperative CEA and postoperative CA24-2 embodied a stronger correlation with OS in which patients had lower OS compared to patients with an increase of either preoperative CEA or postoperative CA24-2 (11 vs. 21 vs. 16 months, $p<0.001$ ). 


\section{Discussion}

In our clinical work, we found that patients with resectable PDAC often have different prognosis after radical resection. Grading of PDAC and surgical guideline are primarily based on imaging results and tumor anatomy. Compared to other cancers with established biomarkers, such as PR, ER and HER-2 in breast cancer [12] and RAS, BRAF, MMR in colon cancer [13], PDAC lacks effective markers to assist in diagnosis, treatment and prognosis. Therefore, this study analyzed clinical data, laboratory tests and pathological diagnosis before and after surgical resection to determine factors that may affect the prognosis of PDAC patients. The study evaluated 29 prognostic factors derived from basic clinical information, pathological finding and laboratory results. All parameters were analyzed by univariate and multivariate COX proportional-hazards model. Information such as age, gender, tumor location and TNM staging were common factors used in analysis of tumor development and prognosis. In this study, we grouped primary tumors into two categories with a cutoff value of $4 \mathrm{~cm}$. This classification has been demonstrated to resemble more closely with prognostic outcome [14]. We also performed analysis of tumors categorized into three groups, and the results did not differ from that of the two categories. In addition, histopathology such as neurological invasion, vascular invasion, carcinoma cell embolism and pancreatic capsule invasion were also included in this study [5, 15]. Laboratory indicators were selected based on convenience and ease of collection. They are commonly used to evaluate patient condition before and after surgery. CA19-9, CEA and CA24-2 are tumor markers that reflect PDAC progression from tumorigenesis, local progression to metastasis [16]. For this reason, tumor markers were collected preoperatively and 1 month after surgery. Serum enzymes such as LDH and ALP were shown to be associated with prognosis in PDAC [17]. Bilirubin and ALB are important indicators of liver metabolism and nutritional status of the patient, which may also influence prognosis [18]. Recent studies have found that preoperative NLR affects prognosis of patients undergoing radical surgery, but there is a lack of reference NLR level [19]. In our study, multivariate COX regression analysis showed that OS in patients that underwent radical resection during early PDAC (stage I and II) had no significant correlation with age, gender, tumor location and TNM stage. There was also no significant correlation between prognosis and the presence of neurological invasion, vascular invasion, vascular tumor thrombus and pancreatic capsule invasion. From serum markers, we found that preoperative CEA and postoperative CA24-2 were independent risk factors of radical resection of PDAC. Other markers, such as CA19-9, LDH, ALD, TBIL, DBIL, ALB, and NLR did not show strong correlation with OS in early stage PDAC patients. It should be noted that a meta-analysis of NLR in PDAC patients revealed no clear cutoff value for NLR, but a cutoff value of 5 was sufficiently stable to evaluate postoperative prognosis $[19,20]$. Therefore, this study used NLR=5 as the cutoff value, and found no statistical difference between $\mathrm{NLR}=2.3[21], \mathrm{NLR}=3$ [22], NLR=4 [23] and NLR=5 on OS.

CA19-9 has been touted as a reliable serum marker for PDAC because of its high sensitivity of $70 \%-90 \%$ and specificity of $90 \%$ for diagnosis [24]. However, in this study CA19-9 did not correlate with postoperative survival of PDAC patients. In addition, due to a lack of Lewis antigen in $6.9 \%$ of patients, the sensitivity of CA19-9 in our test population was only $80 \%$. This phenomenon limited the ability of CA19-9 as an independent biomarker for PDAC prognosis [25]. Patients with resectable PDAC in our study were all in stage I and stage II, and accuracy of CA19-9 can vary with disease stage [26]. Furthermore, one study indicated that the different cutoff values of CA19-9 may have different prognostic efficacy. Therefore, in early stages, CA19-9 may not be an effective biomarker compared to late stages of PDAC [27].

Our study found that preoperative CEA and postoperative CA24-2 were independent markers for poor prognosis of radical PDAC resection. CEA is the second most common serum biomarker used to diagnose PDAC. A meta-analysis estimated that the average sensitivity of CEA for PDAC detection was $44.2 \%(95 \% \mathrm{CI}, 38.5 \%-50.0 \%)$ with an average specificity of $87.5 \%$ (95\%CI, $82.5 \%-91.2 \%$ ) [28]. While some suggested that CEA is inferior to CA19-9 in identifying PDAC, the specificity of the two are similar. A later study supported CEA as a potential biomarker in Lewis-negative PDAC patients, especially in stage I and stage II with better correlation to prognosis [29]. This was consistent with the results of our study. We found that low preoperative CEA correlated with longer survival in PDAC patients after radical surgery. Another marker, CA24-2, is used in conjunction with CA19-9 in early diagnosis of PDAC. Although CA24-2 was found to be less sensitive than CA19-9 in diagnosing PDAC, it was more specific [30]. One study found that pancreatic tumor size of more than $4 \mathrm{~cm}$ showed significantly higher serum CA24-2 level, and cancer invasiveness also correlated positively with CA24-2 level [31]. Interestingly, our study found that postoperative CA24-2 level correlated better with tumor size, lymph node metastasis and PDAC stage than preoperative CA24-2. Our study supported the 
notion that postoperative tumor markers play an important role in resectable PDAC prognosis. When patients showed elevations in both preoperative CEA and postoperative CA24-2 markers, we found that they had lower OS compared with each biomarker alone. This observation suggested that patients with an increase in the combined index will have a worse prognosis. Therefore, combination biomarkers should be used to improve prognostic power.

The current study has several limitations. First, our study evaluated biomarkers for prognosis in the context of early stage PDAC. Established prognostic factors such as CA19-9 [31], LDH, ALP [18] and NLR [20] were mostly used for later stage PDCA and distant metastasis, which was not found to be significant for in ours. It should also be noted that recruited patients were not refrained from receiving other treatments after the initial chemotherapy from our study. Possible treatment variability may have affected the average OS of patients in this study. Postoperative adjuvant therapy therefore should be better controlled in further studies.

\section{Conclusion}

Our study revealed that preoperative CEA and postoperative CA24-2 are biomarkers for prognosis of patients in stage I and II PDAC that underwent radical resection. Elevated CEA and CA24-2 independently and in combination predicted poor survival of patients, where the combination of the two led to worse prognosis. In order to determine the prognosis of patients, tumor markers from before and after surgery should be combined to accurately manage the timing of surgery and adjuvant therapy. A multicenter prospective cohort will be established to investigate the relationship between prognosis and a more comprehensive array of biomarkers in patients at different stages of PDAC undergoing radical surgery.

\section{Abbreviations}

LDH: lactate dehydrogenase; CA19-9: carbohydrate antigen 19-9; CA24-2: carbohydrate antigen 24-2; CA12-5: carbohydrate antigen 12-5; ALB: albumin; PDAC: pancreatic ductal adenocarcinoma; OS: overall survival; ALP: alkaline phosphatase; CEA: carcinoembryonic antigen; ULN: upper limit of normal; LLN: lower limit of normal; HR: hazard ratio; TBIL: total bilirubin; DBIL: direct bilirubin; DSS: disease-specific survival; DFS: disease-free survival; NLR: neutrophils/lymphocytes ratio; CRP: C-reactive protein.

\section{Acknowledgment}

This work was supported by the National Natural Science Foundation of China (grants $81525021,81672431,81672435,81720108028,81772633$, 81702426, 81702427, 81572618, 81802432, 81802433, 81871968 and 81871978). Key Program of Prevention and Treatment of Chronic Diseases of Tianjin (17ZXMFSY001), the programs of Tianjin Prominent Talents, Tianjin Eminent Scholars and Tianjin Natural Science Fund for Distinguished Young Scholar.

\section{Competing Interests}

The authors have declared that no competing interest exists.

\section{References}

1. Ilic M, Ilic I. Epidemiology of pancreatic cancer. World J Gastroenterol. 2016; 22: 9694-705.

2. Kommalapati A, Tella SH, Goyal G, et al. Contemporary Management of Localized Resectable Pancreatic Cancer. Cancers. 2018; 10: 24-39.

3. Kunovsky L, Tesarikova P, Kala Z, et al. The Use of Biomarkers in Early Diagnostics of Pancreatic Cancer. Can J Gastroenterol Hepatol. 2018; 2018: $1-10$.

4. Rajeshkumar NV, Yabuuchi S, Pai SG, et al. Superior therapeutic efficacy of nab-paclitaxel over cremophor-based paclitaxel in locally advanced and metastatic models of human pancreatic cancer. Br J Cancer. 2016; 115: 442-53.

5. Schorn S, Demir IE, Haller B, et al. The influence of neural invasion on survival and tumor recurrence in pancreatic ductal adenocarcinoma - A systematic review and meta-analysis. Surg Oncol. 2017; 26: 105-15.

6. Epstein JD, Kozak G, Fong ZV, et al. Microscopic lymphovascular invasion is an independent predictor of survival in resected pancreatic ductal adenocarcinoma. J Surg Oncol. 2017; 116: 658-64.

7. Hori S, Shimada K, Ino $Y$, et al. Macroscopic features predict outcome in patients with pancreatic ductal adenocarcinoma. Virchows Archiv. 2016; 469: 621-34.

8. Wang Y, Xiao X, Wang T, et al. A Survival Model in Locally Advanced and Metastatic Pancreatic Ductal Adenocarcinoma. J Cancer. 2018; 9: 1301-7.

9. Zhang Y, Yang J, Li H, et al. Tumor markers CA19-9, CA242 and CEA in the diagnosis of pancreatic cancer: a meta-analysis. Int J Clin Exp Med. 2015; 8: 11683-91.

10. Schlitter AM, Jesinghaus M, Jager C, et al. pT but not $\mathrm{pN}$ stage of the 8th TNM classification significantly improves prognostication in pancreatic ductal adenocarcinoma. Eur J Cancer. 2017; 84: 121-9.

11. Tempero MA, Malafa MP, Al-Hawary M, et al. Pancreatic Adenocarcinoma, Version 2.2017, NCCN Clinical Practice Guidelines in Oncology. J Natl Compr Canc Netw. 2017; 15: 1028-61.

12. Bevers TB, Helvie M, Bonaccio E, et al. Breast Cancer Screening and Diagnosis, Version 3.2018, NCCN Clinical Practice Guidelines in Oncology. J Natl Compr Canc Netw. 2018; 16: 1362-89.

13. Benson $A B$, Venook $A P$, Cederquist $L$, et al. Colon Cancer, Version 1.2017, NCCN Clinical Practice Guidelines in Oncology. J Natl Compr Canc Netw. 2017; 15: 370-98

14. Allen PJ, Kuk D, Castillo CF, et al. Multi-institutional Validation Study of the American Joint Commission on Cancer (8th Edition) Changes for $\mathrm{T}$ and $\mathrm{N}$ Staging in Patients with Pancreatic Adenocarcinoma. Ann Surg. 2017; 265: 185-91.

15. Thaler J, Ay C, Mackman N, et al. Microparticle-associated tissue factor activity, venous thromboembolism and mortality in pancreatic, gastric, colorectal and brain cancer patients. J Thromb Haemost. 2012; 10: 1363-70.

16. Chang JC, Kundranda M. Novel Diagnostic and Predictive Biomarkers in Pancreatic Adenocarcinoma. Int J Mol Sci. 2017; 18: 667.

17. Ji F, Fu SJ, Guo ZY, et al. Prognostic value of combined preoperative lactate dehydrogenase and alkaline phosphatase levels in patients with resectable pancreatic ductal adenocarcinoma. Medicine. 2016; 95: e4065.

18. Zhang S, Huang X, Tian $Y$, et al. Clinicopathologic characteristics, laboratory parameters, treatment protocols, and outcomes of pancreatic cancer: a retrospective cohort study of 1433 patients in China. PeerJ. 2018; 6: e4893.

19. Zhou Y, Wei Q, Fan J, et al. Prognostic role of the neutrophil-to-lymphocyte ratio in pancreatic cancer: A meta-analysis containing 8252 patients. Clin Chim Acta. 2018; 479: 181-9.

20. Yang JJ, Hu ZG, Shi WX, et al. Prognostic significance of neutrophil to lymphocyte ratio in pancreatic cancer: a meta-analysis. World J Gastroenterol. 2015; 21: 2807-15. 
21. Szkandera J, Stotz M, Eisner F, et al. External validation of the derived neutrophil to lymphocyte ratio as a prognostic marker on a large cohort of pancreatic cancer patients. PLoS One. 2013; 8: e78225.

22. Teo M, Mohd Sharial MS, McDonnell F, et al. Prognostic role of neutrophil-to-lymphocyte ratio in advanced pancreatic ductal adenocarcinoma: impact of baseline fluctuation and changes during chemotherapy. Tumori. 2013; 99: 516-22.

23. Sugiura $\mathrm{T}$, Uesaka $\mathrm{K}$, Kanemoto $\mathrm{H}$, et al. Elevated preoperative neutrophil-to-lymphocyte ratio as a predictor of survival after gastroenterostomy in patients with advanced pancreatic adenocarcinoma. Ann Surg Oncol. 2013; 20: 4330-7.

24. Duffy MJ, Sturgeon C, Lamerz R, et al. Tumor markers in pancreatic cancer: A European Group on Tumor Markers (EGTM) status report. Ann Surg Oncol. 2010; 21: 441-7.

25. Luo G, Guo M, Jin K, et al. Optimize CA19-9 in detecting pancreatic cancer by Lewis and Secretor genotyping. Pancreatology. 2016; 16: 1057-62.

26. Capello M, Bantis LE, Scelo G, et al. Sequential Validation of Blood-Based Protein Biomarker Candidates for Early-Stage Pancreatic Cancer. J Natl Cancer Inst. 2017; 109. doi: 10.1093/jnci/djw266.

27. Bilici A. Prognostic factors related with survival in patients with pancreatic adenocarcinoma. World J Gastroenterol. 2014; 20: 10802-12.

28. Poruk KE, Gay DZ, Brown K, et al. The clinical utility of CA 19-9 in pancreatic adenocarcinoma: diagnostic and prognostic updates. Curr Mol Med. 2013; 13: 340-51.

29. Luo G, Liu C, Guo M, et al. Potential Biomarkers in Lewis Negative Patients with Pancreatic Cancer. Ann Surg. 2017; 265: 800-5.

30. Gui JC, Yan WL, Liu XD. CA19-9 and CA242 as tumor markers for the diagnosis of pancreatic cancer: a meta-analysis. Int J Clin Exp Med. 2014; 14: 225-33.

31. Chen Y, Gao SG, Chen JM, et al. Serum CA242, CA199, CA125, CEA, and TSGF are Biomarkers for the Efficacy and Prognosis of Cryoablation in Pancreatic Cancer Patients. Cell Biochem Biophys. 2015; 71: 1287-91. 